\title{
Uterine Necrosis following B-Lynch and Gunasheela's Global Stitch Sutures for Primary Postpartum Hemorrhage
}

\author{
${ }^{1}$ BS Punya, ${ }^{2}$ MPA Sailakshmi, ${ }^{3}$ Suhasini Akash, ${ }^{4}$ R Nagarathnamma
}

\begin{abstract}
Uterine atony causing postpartum hemorrhage $(\mathrm{PPH})$ is one of the leading causes of maternal morbidity and mortality. Here by reporting a case of 22-year-old woman who underwent full-term vaginal delivery followed by atonic $\mathrm{PPH}$, for which emergency laparotomy was done and stepwise devascularization along with B-Lynch and Gunasheela's global brace sutures over the uterus. On postoperative day 9, she developed features suggesting sepsis and magnetic resonance imaging (MRI) showed area of avascularity and features of abscess formation in the uterine wall. She underwent subtotal hysterectomy and histopathology showed uterine necrosis.
\end{abstract}

Keywords: B-Lynch suture, Gunasheela's global stitch, Postpartum hemorrhage, Uterine necrosis.

How to cite this article: Punya BS, Sailakshmi MPA, Akash S, Nagarathnamma R. Uterine Necrosis following B-Lynch and Gunasheela's Global Stitch Sutures for Primary Postpartum Hemorrhage. J Med Sci 2015;1(2):38-40.

Source of support: Nil

Conflict of interest: None

\section{INTRODUCTION}

Hemorrhage remains a major cause of maternal morbidity and mortality in the developed and developing world. Fourteen million cases of postpartum hemorrhage (PPH) occur worldwide each year, with a case-fatality rate of $1 \%$, this is a total of 140,000 women, (one every 4 minutes). ${ }^{1}$ Postpartum hemorrhage has been defined as blood loss in excess of $500 \mathrm{ml}$ in a vaginal birth and in excess of $1000 \mathrm{ml}$ in a cesarean delivery. For clinical purposes, any blood loss that has the potential to produce hemodynamic instability $(10 \%$ change in the hematocrit) should be considered PPH. Primary (immediate) PPH occurs within the first 24 hours after delivery. Approximately, $70 \%$ of immediate PPH cases are due to uterine atony. Secondary (late) PPH occurs between 24 hours after delivery of the infant and 6 weeks postpartum.

\footnotetext{
${ }^{1}$ Postgraduate, ${ }^{2,3}$ Professor, ${ }^{4}$ Professor and Head

${ }^{1-4}$ Department of Obstetrics and Gynecology, RajaRajeswari Medical College and Hospital, Bengaluru, Karnataka, India

Corresponding Author: BS Punya, Postgraduate, Department of Obstetrics and Gynecology, RajaRajeswari Medical College and Hospital, Bengaluru, Karnataka, India, Phone: 9739460265, e-mail: elijahwood.puns@gmail.com
}

Postpartum hemorrhage is responsible for around $25 \%$ of maternal mortality worldwide (WHO, 2007), reaching as high as $60 \%$ in some countries. Postpartum hemorrhage can also be a cause of long-term severe morbidity, and approximately $12 \%$ of women who survive PPH will have severe anemia (Abou-Zahr, 2003; WHO, 2006). Additionally, women who have severe PPH and survive (near misses) are significantly more likely to die in the year following the PPH (Impact International, 2007). Successful techniques using compression sutures to control PPH have been described, in some cases using a combination of techniques. Here, we describe a case in which compression sutures to control primary PPH were complicated by subsequent myometrial necrosis.

\section{CASE REPORT}

A 22-year-old patient, primigravida with term gestation was admitted to our labor room with history of 6 hours of leaking per vaginal (PV). She had an uneventful antenatal period. Tablet misoprost- $50 \mu \mathrm{g}$ was given orally and monitored followed by normal vaginal delivery, prophylactic injection methergine was given after the delivery of the baby. Uterus was well contracted immediately after the delivery. Slow trickling of blood was noticed PV after half an hour post delivery, vaginal exploration was done and was made sure that it was bleeding from within the uterus and not from the cervix or the episiotomy wound, hence, traumatic PPH was ruled out.

Fundal massage was given, followed by injection syntocinon, $10 \mathrm{IU}$ IM and $20 \mathrm{IU}$ in $500 \mathrm{ml}$ ringer lactate (RL), injection prostodin $250 \mu \mathrm{g}$, 4 doses were given, followed by $\mathrm{T}$ misoprost $600 \mu \mathrm{g}$ per rectum (PR). Uterus continued to be atonic and patient became pulseless and was immediately shifted to operation room with bimanual compression, emergency laparotomy procedure was done. Intraoperatively, uterus was flabby and atonic. Stepwise devascularization was done, bilateral ovarian arteries ligated followed by ligation of uterine arteries. Since the uterus continued to be atonic, B-Lynch brace suture was applied followed by 3 tiers of Gunasheela's global sutures using vicryl no. 1,90 mm sutures, after which hemostasis was achieved. 


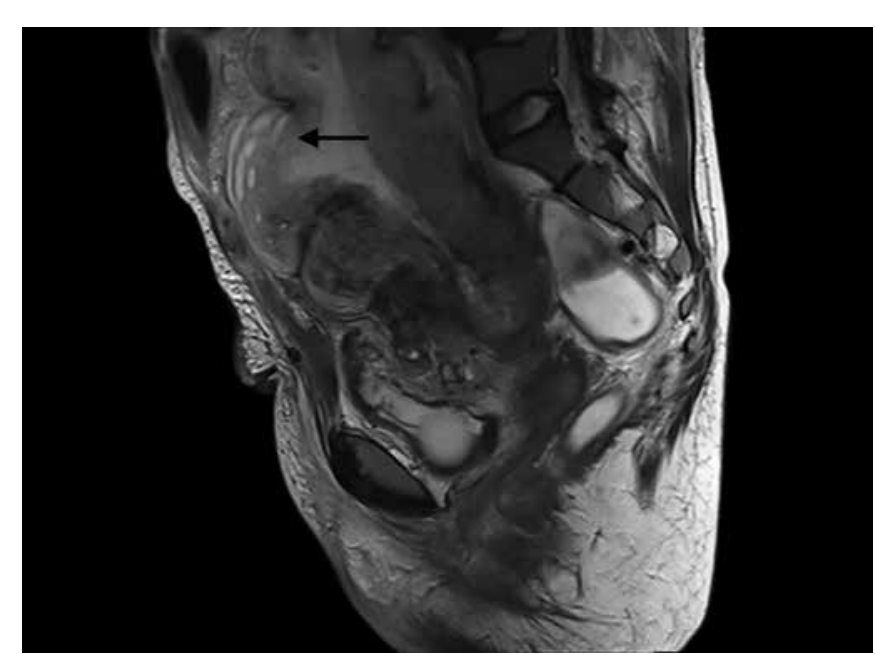

Fig. 1: Magnetic resonance image showing a suspected area of necrosis in the uterus (arrow mark)

Postoperatively, patient was on ventilator for 2 days and then extubated, all together estimated blood loss was more than 1.6 L, four packed red blood cells (PRBCs), four fresh frozen plasmas (FFPs) and two platelets and two cryoprecipitate were given until her hemogram and coagulation profiles came within normal limits. Patient developed fever and pain in abdomen on day 4 of the postoperative and an ultrasound scan revealed splenomegaly, ascites and left-sided pleural effusion, uterine echotexture was not well made out. Multiplanar, multisequence magnetic resonance imaging (MRI) scanning was performed, which showed an avascular area in the anterior wall of the uterus and features suggesting of formation of abscess in the uterus (Fig. 1). Following the imaging, hysterectomy was recommended because of the persisting risk of sepsis and hemorrhage. The patient was initially reluctant to undergo further surgery, but when symptoms continued, she got discharged against medical advice and went to another tertiary care center at Bengaluru, on postoperative day 10 , where she underwent laparotomy, blood stained ascitic fluid and an inflamed uterus were found, subtotal hysterectomy was done and the patient got discharged on day 9 with good postoperative recovery, no complications.

Histological examination confirmed inflamed uterus with necrosis of a part of myometrium in the anterior wall of the uterus. Patient has been followed up for a year now, no complications.

\section{DISCUSSION}

Major postpartum hemorrhage is a significant obstetric emergency requiring prompt management to prevent maternal mortality. The B-Lynch technique of a 'belt and braces' uterine compression suture was described in 1997 as an alternative to cesarean hysterectomy, pelvic vascular surgery or interventional radiological procedures (such as uterine artery embolization) in the case of major $\mathrm{PPH}^{2}{ }^{2}$ Several other compression sutures have been described and they act by direct application of pressure on the placental bed and by reducing the blood to uterus. Isolated complications of conservative surgical management of PPH, including compression and ligation techniques have been reported. Ischemic necrosis of uterus have been described by Treolar E et al, ${ }^{3}$ Akoury and Sherman, ${ }^{4}$ Joshi and Shrivastava, ${ }^{5}$ Gottelieb et al. ${ }^{6}$

Imaging played a very important role in aiding our diagnosis, although ultrasound is the first line of imaging, alternative modalities must be considered in unusual cases like this. Magnetic resonance imaging scanning was a useful adjunct in this case and its use has previously been described in imaging uterine necrosis. ${ }^{7}$ It has been suggested that in this case an incorrectly placed B-Lynch suture or combining it with the Gunasheela's global suture could have interrupted the uterine arterial blood flow to the myometrium and possibly explain the mechanism of uterine infarction.

\section{CONCLUSION}

While uterine compression sutures are useful in the containment of PPH due to uterine atony and have prevented many immediate hysterectomies, complications are to be kept in mind before adopting them for routine use. Data on efficacy and safety of these compression suturing techniques are mainly limited to the small case series reported by the proponents themselves, and long-term follow-up information is still lacking. A randomized trial evaluating and comparing the efficacy of the procedures that can be used for the treatment of primary PPH is clearly required. More than all simulator aided workshops and hands-on trainings must be conducted about these compression sutures and PPH. Judicious use of compression sutures has to be practiced. Other contingent protocols should be available, should compression sutures fail to control the hemorrhage. The combined or sequential use of compression sutures with other treatment modalities, such as balloon tamponade, pelvic devascularization or radiological embolization, may help to increase the success rate, and should be explored further.

\section{REFERENCES}

1. Estimates developed by WHO, UNICEF and UNFPA. Geneva: Department of Reproductive Health and Research, World Health Organization; 2004. WHO. Maternal Mortality in 2000.

2. B-Lynch C, Coker A, Lawal AH, Abu J, Cowen MJ. The B-Lynch surgical technique for the control of massive 
postpartum haemorrhage: an alternative to hysterectomy? Five cases reported. Br J Obstet Gynaecol 1997;104:372-376.

3. Treolar E', Anderson R, Andrews H, Bailey J. Uterine necrosis following B-Lynch suture for primary postpartum haemorrhage. BJOG 2006;113:486-488.

4. Akoury $\mathrm{H}$, Sherman C. Uterine wall partial thickness necrosis following combined B-Lynch and Chi square sutures for the treatment of primary postpartum hemorrhage. JOGC 2008;30(5):421-424.
5. Joshi VM, Shrivastava M. Partial ischemic necrosis of the uterus following uterine brace compression suture. BJOG 2004;111:279-280.

6. Gottelieb AG, Panipati S, Davis KM, Gibbs RS. Uterine necrosis: a complication of uterine compression sutures. Obstet Gynaecol 2008 Aug;112(2 Pt 2):429-431.

7. Pretorius ES, Siegelman ES, Tureck R, Deerlin VV. Uterine necrosis after caesarean delivery seen on MR imaging. Am J Roentgenol 1997;168:849-850. 